\title{
Gestational diabetes (GDM) does not predict large birthweight or perinatal death in a relatively untreated population in Uganda; a prospective observational cohort study.
}

Jack Milln ${ }^{1}$, Betty Nakabuye ${ }^{2}$, Barnabas Natamba ${ }^{1}$, Isaac Sekitoleko ${ }^{1}$, Michael Mubiru ${ }^{1}$, Arthur Namara ${ }^{1}$, Samuel Tumwesigire ${ }^{1}$, Tino Salome ${ }^{1}$, Mandy Wilja ${ }^{1}$, Ayoub Kakande ${ }^{1}$, Brian Agaba ${ }^{2}$, Faridah Nansubuga ${ }^{3}$, Daniel Zaake ${ }^{3}$, Ben Ayiko ${ }^{4}$, Herbert Kalema ${ }^{5}$, Sarah Nakubulwa $^{6}$, Musa Sekikubo ${ }^{7}$, Annettee Nakimuli ${ }^{7}$, Emily $\mathrm{Webb}^{8}$, and Moffat Nyirenda ${ }^{1}$

${ }^{1}$ MRC/UVRI Uganda Research Unit On AIDS

${ }^{2}$ Lubaga Hospital

${ }^{3}$ Nsambya Hospital

${ }^{4}$ Entebbe General Hospital

${ }^{5}$ Masaka Regional Referral Hospital

${ }^{6}$ Makerere University College of Health Sciences

${ }^{7}$ Makerere University and Mulago National Referral Hospital

${ }^{8} \mathrm{LSHTM}$

May 11, 2020

\begin{abstract}
Objective: To determine whether hyperglycaemia in the gestational diabetes (GDM) range independently predicts adverse pregnancy outcomes in Uganda. Design: Prospective observational cohort study. Setting:Five major hospitals in urban/semi-urban central Uganda. Sample:237 women with gestational diabetes, 2,641 normoglycaemic controls. Methods:Women were screened with oral glucose tolerance test (OGTT) at 24-28 weeks of gestation. Cases of GDM were identified (WHO 2013 diagnostic criteria) and received standard care. Data was collected on maternal demographics, anthropometrics, prenatal management, umbilical cord c-peptide levels, and pregnancy outcomes. Participants with diabetes in pregnancy (DIP) were excluded from the analysis. Outcomes:Primary outcomes: Birthweight large for gestational age (LGA; >90th centile) and perinatal death. Secondary outcomes: Caesarean delivery, preterm birth $<37$ weeks, umbilical cord c-peptide concentration $>90$ th centile $(>1.35$ $\mathrm{mcg} / \mathrm{L}$ ), and neonatal admission. Results:Women with GDM had a median of only two glucose measurements recorded in third trimester, and only one fifth received therapeutic management (mostly metformin, one participant received insulin). GDM was not independently associated with LGA (adjusted odds ratio, aOR 1.12; 95\% CI 0.81-1.56) or perinatal death (aOR 0.66; 95\% CI 0.26-1.66), but increased the risk of Caesarean delivery. Mid-gestational BMI of $>30 \mathrm{~kg} / \mathrm{m} 2$ was strongly associated with LGA, and mean arterial pressure $>90 \mathrm{mmHg}$ was the strongest predictor of perinatal death. Conclusions:Even without active management, GDM was not associated with large birthweight or perinatal death in this population. Interventions that target blood pressure and obesity are likely to be more beneficial in improving LGA and perinatal mortality, than management of GDM. Funding:Medical Research Council Keywords:Gestational diabetes, Africa
\end{abstract}

GDM does not predict large birthweight or perinatal death in a relatively untreated population in Uganda; a prospective observational cohort study.

Jack Milln (MBBS $)^{1,2 *}$ and Betty Nakabuye (MMED) ${ }^{3,4}$, Barnabas Natamba $(\mathrm{PhD})^{1}$, Isaac Sekitoleko $(\mathrm{MSc})^{1}$, Michael Mubiru (BSc) ${ }^{1}$, Arthur Araali Namara $(\mathrm{MSc})^{1}$, Samuel Tumwesigire $(\mathrm{MBchB})^{1}$, Salome 
Tino $(\mathrm{MPH})^{1}$, Mandy Mirembe $(\mathrm{MSc})^{1}$, Ayoub Kakande $(\mathrm{MSc})^{1}$, Brian Agaba (MMED) ${ }^{3}$, Faridah Nansubuga (MMED) ${ }^{5}$, Daniel Zaake (MMED) $)^{5}$, Ben Ayiko (MMED) $)^{6}$, Herbert Kalema (MMED) ${ }^{7}$, Sarah Nakubulwa $(\mathrm{PhD})^{8,9}$, Musa Sekikubo $(\mathrm{PhD})^{8,9}$, Annettee Nakimuli $(\mathrm{PhD})^{8,9}$, Emily L Webb $(\mathrm{PhD})^{10}$, Moffat J Nyirenda $(\mathrm{PhD})^{1,10}$

1 Non-Communicable Diseases Theme, Medical Research Council/Uganda Virus Research Institute and London School of Hygiene and Tropical Medicine (MRC/UVRI \& LSHTM) Uganda Research Unit, Plot 51-59, Nakiwogo Road, P. O. BOX 49, Entebbe, Uganda.

${ }^{2}$ Department of Endocrinology and Diabetes, Queen Mary University of London, Mile End Road, London, UK.

${ }^{3}$ Uganda Martyrs Hospital, Lubaga, Kampala, Uganda.

${ }^{4}$ School of Public Health, Makerere University College of Health Sciences, Kampala, Uganda

${ }^{5}$ St. Francis Hospital, Nsambya, Kampala, Uganda

${ }^{6}$ Entebbe Regional Referral Hospital, Entebbe, Uganda

${ }^{7}$ Masaka Regional Referral Hospital, Masaka, Uganda

8 Department of Obstetrics and Gynaecology, School of Medicine, Makerere University College of Health Sciences, Kampala, Uganda

${ }^{9}$ Kawempe Specialized National Referral Hospital, Kampala, Uganda

${ }^{10}$ London School of Hygiene and Tropical Medicine (LSHTM), London, UK

* Corresponding author

\section{Corresponding author:}

Dr Jack Milln

MRC/UVRI \& LSHTM Uganda Research Unit, Plot 51-59, Nakiwogo Road, P. O. BOX 49, Entebbe, Uganda.

Tel.: +256 793 392872. No fax available

jackmilln@doctors.org.uk

Running title: Pregnancy outcomes associated with GDM in Uganda

ABSTRACT - as per BJOG template, 250 words (currently 248)

Objective: To determine whether hyperglycaemia in the gestational diabetes (GDM) range independently predicts adverse pregnancy outcomes in Uganda.

Design: Prospective observational cohort study.

Setting: Five major hospitals in urban/semi-urban central Uganda.

Sample: 237 women with gestational diabetes, 2,641 normoglycaemic controls.

Methods: Women were screened with oral glucose tolerance test (OGTT) at 24-28 weeks of gestation. Cases of GDM were identified (WHO 2013 diagnostic criteria) and received standard care. Data was collected on maternal demographics, anthropometrics, prenatal management, umbilical cord c-peptide levels, and pregnancy outcomes. Participants with diabetes in pregnancy (DIP) were excluded from the analysis.

Outcomes: Primary outcomes: Birthweight large for gestational age (LGA; $>90^{\text {th }}$ centile) and perinatal death. Secondary outcomes: Caesarean delivery, preterm birth $<37$ weeks, umbilical cord c-peptide concentration $>90^{\text {th }}$ centile $(>1.35 \mathrm{mcg} / \mathrm{L})$, and neonatal admission. 
Results: Women with GDM had a median of only two glucose measurements recorded in third trimester, and only one fifth received therapeutic management (mostly metformin, one participant received insulin).

GDM was not independently associated with LGA (adjusted odds ratio, aOR 1.12; 95\% CI 0.81-1.56) or perinatal death (aOR 0.66; 95\% CI 0.26-1.66), but increased the risk of Caesarean delivery. Mid-gestational BMI of $>30 \mathrm{~kg} / \mathrm{m}^{2}$ was strongly associated with LGA, and mean arterial pressure $>90 \mathrm{mmHg}$ was the strongest predictor of perinatal death.

Conclusions: Even without active management, GDM was not associated with large birthweight or perinatal death in this population. Interventions that target blood pressure and obesity are likely to be more beneficial in improving LGA and perinatal mortality, than management of GDM.

Funding: Medical Research Council

Keywords: Gestational diabetes, Africa

Tweetable abstract: 'GDM does not predict large birthweight or perinatal death in Uganda, despite low intensity management; interventions targeting blood pressure and obesity are likely more beneficial'

INTRODUCTION - 400 words for BJOG (currently 392)

Hyperglycaemia first detected in pregnancy (HIP) is currently classified, based on $75 \mathrm{~g}$ oral glucose tolerance test (OGTT), as diabetes in pregnancy (DIP) or gestational diabetes mellitus (GDM), a milder form. ${ }^{1}$ While the association between DIP and poor pregnancy outcomes is clear ${ }^{2}$, the contribution of milder levels of hyperglycaemia in the GDM range (fasting glucose 5.1-7.0 mmol/L, 2-hour OGTT glucose level 8.5-11.0 $\mathrm{mmol} / \mathrm{L}$ ) has long been debated. Recently, the HAPO study showed hyperglycaemia within the GDM range was linearly associated with adverse pregnancy outcomes, notably large birthweight $\left(>90^{\text {th }}\right.$ centile). ${ }^{3}$ This has led to recent tightening of international diagnostic criteria for GDM in order to capture women with milder derangements in glucose control. ${ }^{4}$ Subsequently, some studies have shown that treating such mild levels hyperglycaemia is associated with modest improvement in outcomes, although in most cases this required intensive interventions such as insulin use, multiple daily self-monitoring of blood glucose ${ }^{5}$ or induction of labour. ${ }^{6,7}$

Both the HAPO and subsequent intervention studies were largely undertaken in high-income countries, and the benefits of these screening and management approaches may not necessarily directly translate to other populations, particularly those in resource poor settings, such as sub-Saharan Africa (SSA). The International Diabetes Federation (IDF) estimates that 1 in 6 women in the African region may be affected by hyperglycaemia in pregnancy, raising the profile of GDM on the international development agenda around NCD prevention and management. ${ }^{8}$ However, in most countries in SSA, screening and treatment of HIP is not common, and there is paucity of studies on screening, treatment and obstetric outcomes of HIP; loose recommendations are largely based on external evidence, or on small studies with heterogeneous methodologies and criteria. ${ }^{9-11}$ In these resource constrained settings, there is a clear need to develop optimal screening and management strategies that will identify and target women with HIP who are at significant risk of clinically relevant adverse obstetric outcomes.

The aim of this study was therefore to critically assess whether hyperglycaemia in the GDM range, obtained by OGTT, independently predicted poor pregnancy outcomes, particularly large birthweight (defined as $>90^{\text {th }}$ centile) and perinatal death, in women living in urban and peri-urban Uganda. Perinatal death was chosen as an outcome in our study due to the higher perinatal mortality rate in the SSA setting, compared to sites in the HAPO study. Other variables/exposures with potential to impact pregnancy outcomes were also explored.

\section{METHODS}

\section{Setting}

This observational cohort study recruited women attending antenatal care at five major hospitals in urban 
and peri-urban areas of central Uganda between $13^{\text {th }}$ June 2018 and $31^{\text {st }}$ October 2019. Three are public facilities managed by the Uganda Ministry of Health, and two are private-not-for-profit hospitals managed by the Uganda Catholic Medical Services Bureau. Uganda is a low-income country in East Africa with an annual GDP of $\$ 643$ per capita ( $\$ 1.76$ per day). ${ }^{12}$ Whilst great progress has been made in recent decades, the maternal mortality rate remains high (368 per 100,000 live births), as does the perinatal mortality rate (42 per 1,000 births). ${ }^{13,14}$

\section{Participants}

Pregnant women were eligible to participate if they were 18 years or older and between 24 and 28 weeks of gestation calculated using date of last menstrual period and/or earliest obstetric ultrasound scan where available. Women were excluded if they had one or more of the following exclusion criteria: known diagnosis of diabetes, significant medical comorbidity (such as heart failure, renal disease, severe anaemia), multiple pregnancy, inability to provide informed consent, or plans to deliver at a non-study facility.

Women were approached in the antenatal clinic by the research team and screened for inclusion and exclusion criteria.

At recruitment, standardised questionnaires were used to collect data on socio-demographic and lifestyle factors (including age, level of education, smoking status and alcohol use). Questionnaires also covered family, medical (including HIV status) and reproductive history (parity, gravidity and complications in prior pregnancies). Weight, height, and mid-upper-arm circumference were measured using calibrated Seca scales, stadiometers, and flexible tape measures. After 30 minutes of rest, three seated blood pressure measurements, with 5 minutes rest in between, were collected on the right arm using portable sphygmomanometers (OMRON-Healthcare-Co HEM-7211-E-Model-M6; Kyoto, Japan). We used the mean of the last two blood pressure readings.

\section{Oral glucose tolerance test.}

Participants underwent a standard oral glucose tolerance test after an overnight fast of at least 8 hours. A fasting venous blood glucose was collected, and participants were then given $82.5 \mathrm{~g}$ glucose monohydrate (equivalent to $75 \mathrm{~g}$ anhydrous glucose) dissolved in $250 \mathrm{ml}$ of water. Repeat venous blood samples were taken at 60 and 120 minutes. Samples were immediately centrifuged at study sites and plasma stored on ice. All samples were analysed centrally at the MRC/UVRI and LSHTM Clinical and Diagnostics Laboratory in Entebbe, within 4 hours of collection, or stored at $-80^{\circ} \mathrm{C}$ for subsequent analysis.

\section{Diagnosis and management of women with hyperglycaemia in pregnancy}

HIP was diagnosed according to WHO 2013 criteria as GDM: fasting glucose [?] 5.1 and $<6.9 \mathrm{mmol} / \mathrm{L}$ or 1-hour glucose [?]10.0mmol/L or 2-hour glucose [?] 8.5 and $<11.0 \mathrm{mmol} / \mathrm{L}$; and, DIP: fasting glucose [?]7.0mmol/L or 2-hour glucose [?]11.1mmol/L. Women with hyperglycaemia in pregnancy were notified and invited to meet the local obstetric team for further management. A summary of local management practices conducted prior to the study is provided in the Supplementary Appendix (Table S1). Clinicians were provided with a basic treatment protocol based on the FIGO pragmatic guide for diabetes antenatal care in the resource-limited setting. ${ }^{15}$ Antenatal management was recorded with a standardised proforma by the obstetric team at each study site including the number of antenatal visits, fasting capillary glucose values, treatment administered, and third trimester ultrasound scan results. This study was aimed at examining the association of GDM, specifically, and pregnancy outcomes; women with DIP were therefore excluded from subsequent analyses. For this study, we considered GDM as 'controlled', 'partially controlled' or 'uncontrolled' if the mean of the two fasting capillary blood glucose values prior to delivery were $<5.1$ $\mathrm{mmol} / \mathrm{L}, 5.1-7.0 \mathrm{mmol} / \mathrm{L}$, or $>7.0 \mathrm{mmol} / \mathrm{L}$ respectively. If cases were not seen in the antenatal clinic or only had one fasting capillary glucose result, they were coded as 'unknown'.

\section{Collection of outcome data}

Maternal and neonatal outcomes were extracted from mothers' records at the time of delivery and recorded 
by midwives. These included: maternal antenatal complications (hypertensive disorders of pregnancy, poly/oligohydramnios), delivery complications (prolonged labour, ruptured uterus, shoulder dystocia), mode of delivery, birthweight and gestational age of neonate. Hypertensive disorders of pregnancy included gestational hypertension, pre-eclampsia, and eclampsia. Macrosomia and low-birthweight were defined as birthweight $>4 \mathrm{~kg}$ and $<2.5 \mathrm{~kg}$, respectively. Further data were recorded at the time of discharge from hospital and included neonatal complications (neonatal admission, hypoglycaemia, jaundice) and neonatal death. Neonatal admission was defined as formal admission of the neonate to the special baby unit for observation and/or treatment beyond routine neonatal care. Umbilical cord blood, to estimate serum c-peptide concentration, was obtained at the time of delivery by midwives; samples were immediately centrifuged at study sites, stored on ice, and analysed at the MRC/UVRI \& LSHTM Uganda Research Unit central laboratory.

\section{Definition of primary and secondary outcomes.}

The primary outcomes were birthweight $>90^{\text {th }}$ centile using the INTERGROWTH-21 population standards ${ }^{16}$ and perinatal death (stillbirth $>24$ weeks and neonatal death $<28$ days). Secondary outcomes were Caesarean delivery, preterm birth $<37$ weeks, umbilical cord serum c-peptide concentration $>90^{\text {th }}$ centile, and neonatal admission. Umbilical cord c-peptide concentration is an indirect marker of fetal hyperinsulinaemia and surrogate marker of clinical neonatal hypoglycaemia. ${ }^{3,7}$ The $90^{\text {th }}$ centile was defined on the basis of the participants included in the final analysis.

\section{Statistical analysis.}

Participants' baseline characteristics were summarised using means, medians, standard deviations, and interquartile ranges for continuous variables where applicable. Categorical data were summarized using numbers and proportions. We used Pearson's chi-square and Fisher's exact tests to assess the associations between GDM and maternal and neonatal outcomes, and present crude odds ratios (ORs).

Participants with DIP were excluded from the multivariate analysis. Univariable and multivariable logistic regression was used to investigate the effect of GDM, as a categorical variable, and OGTT blood glucose concentrations as continuous variables, for each of the primary and secondary outcomes. These were adjusted for potential confounders i.e. study site, mid-gestational BMI, maternal height, maternal age, parity, mean arterial pressure, HIV status, and previous macrosomia. These final adjust models are presented as odds ratios and their $95 \%$ confidence interval, as per the HAPO Study. ${ }^{3}$

For continuous variable analysis, odds ratios were calculated for every 1-SD increase in the fasting, 1-hour and 2-hour plasma glucose concentrations, as per the HAPO study. ${ }^{3}$ All analyses were conducted in STATA 15.1 (College Station, Texas).

\section{Ethical approval}

This research project was approved by the research and ethics committee of the Uganda Virus Research Institute (approval GC/127/19/04/625) and the Uganda National Council for Science and Technology (approval HS2340). All participating women gave informed written consent. A minimal compensation for participants' time and meal after undergoing the OGTT was provided.

\section{RESULTS}

\section{Maternal characteristics}

The study enrolled a total of 3852 participants. Of these, 2917 participants were included in the analysis. The remainder of the participants $(n=935)$ were excluded because they either had incomplete laboratory and/or outcome data (Figure 1). Excluded participants had similar baseline characteristics as those included in the final analysis. Of those who were included in the analysis, 2,641 were normoglycaemic, 237 had hyperglycaemia in the GDM range, and 39 had hyperglycaemia in the DIP range. Data from participants with DIP were excluded from subsequent analysis but are displayed in the Supplementary Appendix (Tables S2 and S3). 


\section{Figure 1 insert here.}

The characteristics of participants are displayed in Table 1. All women in the study were of black ethnicity. The mean age of participants was 27.0 years, the mean maternal BMI at time of OGTT was $27.7 \mathrm{~kg} / \mathrm{m}^{2}$. Nearly a third of women were primigravid $(915 / 2917 ; 31.4 \%)$, around one third were primiparous $(943 / 2917$; $32.2 \%$ ), and approximately one third were multiparous (two or more children: 1059/2917 36.3\%).

Compared to normoglycaemic women, those with GDM were older, more likely to attend a private facility, and more likely to be obese, multiparous, and have a history of diabetes in the family or previous macrosomia.

Table 1 insert here.

\section{Management}

Women diagnosed with GDM were seen around twice in the diabetes antenatal clinic after their diagnosis (median, 2; IQR, 1-4). Approximately half of women with GDM (49.8\%) received lifestyle advice only, $19.4 \%$ received low dose metformin ([?]1g/day), $1.7 \%$ received higher dose metformin $(>1 \mathrm{~g} /$ day) and one woman received insulin as a twice daily premixed preparation. Only one woman, who was treated with insulin, performed home self-monitoring of blood glucose. Half of the women with GDM (49.8\%) had $<2$ fasting glucose measurements documented during antenatal follow-up. Of the remaining, around half appeared controlled (mean of 2 fasting blood glucose values $<5.1 \mathrm{mmol} / \mathrm{L}$ ) and half appeared either partially controlled (mean of 2 fasting blood glucose values 5.1-7.0 mmol/L) or uncontrolled (mean of 2 fasting blood glucose values $>7.0 \mathrm{mmol} / \mathrm{L})$. Few women $(15.6 \%)$ had documentation of a third trimester ultrasound scan. A summary of the diabetic management of women with GDM is shown in the Supplementary Appendix (Table S4).

\section{Maternal outcomes}

There was no significant difference in poly/oligohydramnios between women who had GDM and those who were normoglycaemic. Participants within the GDM group had higher risk of hypertensive disorders in pregnancy compared to the normoglycaemic participants $(8.0 \%$ Vs $3.8 \%$; p-value $<0.01)$. Similarly, a higher proportion of women with GDM underwent Caesarean delivery as compared to normoglycaemic participants (39.7\% Vs 28.7\%; p-value <0.01). Approximately three quarters $(73.2 \%)$ of Caesarean deliveries were coded as 'Emergency' rather than 'Elective', with similar distribution among the GDM and normoglycaemic groups. There were no significant differences in other maternal delivery complications between the groups (Table 2). There was one maternal death, in the GDM group, due to intrapartum haemorrhage from a ruptured uterus secondary to obstructed labour.

\section{Neonatal outcomes}

There was no difference in the median umbilical cord c-peptide concentration or proportion $>90^{\text {th }}$ centile between babies that were born to GDM mothers and normoglycaemic mothers. Similarly, the rate of clinical neonatal jaundice was similar between the groups. However, more cases of neonatal hypoglycaemia were recorded in the GDM group, compared to babies born to normoglycaemic mothers (1.8\% Vs 0.4\%; p-value 0.02). Babies born to mothers with GDM were on average $47.0 \mathrm{~g}$ larger than those born to normoglycaemic participants, but this was not statistically significant. Similarly, there were no differences in the proportion of babies born large for gestational age $(25.0 \%$ Vs $20.4 \%$; p-value 0.15$)$, or macrosomic, small for gestational age, or low birthweight. Gestational age at delivery, proportion of babies born preterm, proportion of babies admitted to the special baby unit and their median length of stay were similar between babies that were born to GDM mothers and normoglycaemic mothers. There was no significant difference detected in perinatal mortality rates between GDM and normoglycaemic pregnancies (2.11\% Vs 2.8\%; p-value 0.68). Neonatal outcomes are displayed in Table 2 .

Table 2 insert here.

Associations with primary and secondary outcomes 
In both the crude and adjusted analysis, GDM was not associated with any of our primary or secondary outcomes aside from Caesarean delivery (crude OR, 95\% CI; 1.64, 1.23-2.18; adjusted OR, 95\% CI; 1.36, $1.02-1.82)$.

In adjusted models, the strongest predictors of LGA were mid-gestational BMI $>30 \mathrm{~kg} / \mathrm{m}^{2}$ (OR 2.21; 95\%CI 1.70-2.90) and previous macrosomia (OR 2.41; 95\% CI 1.83-3.19), and the strongest predictors of perinatal death were mean arterial pressure $>90 \mathrm{mmHg}$ (OR 2.49; 95\% CI 1.41-4.39) and positive HIV status (OR 2.49; 95\% CI 0.96-6.47). The elements of the adjusted models are displayed in the Supplementary Appendix (Table S5).

With the exception of Caesarean delivery, no association was found when outcomes were analysed against OGTT glucose concentrations as continuous variables (Table 3).

Table 3 insert here.

DISCUSSION - 1200 words for BJOG (currently 1144)

\section{Main findings}

Our study in Ugandan women shows that GDM, even without intensive management, did not significantly increase in the risk of major outcomes that are typically seen with overt diabetes. In contrast, obesity and high mean arterial pressure were associated with excessive birthweight and perinatal death, respectively.

\section{Strengths and limitations}

To our knowledge this is the largest and most robust prospective investigation of pregnancy outcomes in GDM in Africa. We made efforts to ensure that it is as representative as possible by recruiting from both public and private facilities. We made use of a rigid study protocol to collect detailed data, utilised high quality central laboratory for sample analyses, employed up-to-date diagnostic criteria.

In our study, women diagnosed with GDM were referred to be managed by their clinicians, rather than through a study protocol; the intensity of treatment was therefore likely to be variable and not under study control, which may have influenced outcomes. Similarly, for some of the outcomes (such as pre-eclampsia, poly/oligohydramnios) we relied on healthcare records rather than active investigation by the study team. We did not have data regarding previous operative delivery and so could only report Caesarean delivery, rather than primary Caesarean delivery. The study was performed in urban and peri-urban central Uganda which may reduce generalisability to rural populations.

\section{Interpretation (in light of other evidence)}

Sub-Saharan Africa is undergoing a rapid demographic and nutritional transition associated with rapid urbanisation. With an emerging epidemic of type 2 diabetes across the continent, hyperglycaemia in pregnancy is likely to pose a major health challenge in this region in the future. Screening and management of HIP are resource intensive. To give some context, the per capita health expenditure in Uganda is $\$ 37.6$ per year, compared to $\$ 3,958$ per year in the UK (2016 figures). ${ }^{17}$ Although there are no cost-effectiveness studies for GDM screening from Africa, a study from India estimated a cost of $\$ 1626$ per life year gained. ${ }^{18}$ Costs of management, often requiring insulin therapy and ambulatory capillary glucose monitoring, are absorbed by women and are currently prohibitively expensive to most. ${ }^{5}$ In order to prioritise resources efficiently, it is therefore imperative to generate local evidence to ensure only women most at risk of clinically relevant adverse pregnancy outcomes are identified and treated.

In our study, the milder form of hyperglycaemia, GDM, was not associated with birthweight $>90^{\text {th }}$ centile. Whilst having larger babies is not a disorder in itself, it may lead to obstructed labour and operative delivery, and is therefore commonly used as a marker of perinatal adversity. This may have particularly grave consequences in the low-income setting where antenatal and perinatal care is suboptimal. ${ }^{19,20}$ Additionally, women in sub-Saharan Africa are already more prone to obstructed labour due to cephalopelvic disproportion. ${ }^{21-24}$ While the HAPO study described a clear association between glycaemia and LGA, our 
study did no show this relationship. The reasons for this discrepancy are unclear, but they may relate to sample size. Nonetheless, if there is association within our population, this is unlikely to have significant clinical relevance. ${ }^{25}$

However, in accord with HAPO (which revealed that BMI was a stronger predictor than glycaemia ${ }^{26}$ ), our data show that mid-gestational BMI was a strong predictor of LGA. In the HAPO study, $78 \%$ of LGA babies were born to mothers with normal glucose tolerance, ${ }^{27}$ whilst in our study nearly $90 \%$ of LGA infants were from normoglycaemic mothers. These data underscore the contribution of other (non-glucose) physiological determinants of fetal growth. While there are few studies that have explored associations between HIP and birthweight in Africa, ${ }^{11}$ those that do exist also point towards BMI as a more reliable predictor of LGA than glycaemia. ${ }^{9,28,29}$

GDM was also not associated with an increased risk of perinatal mortality. However, we observed few perinatal deaths, and the study may not have been sufficiently powered for this outcome. The background rate of perinatal mortality in Uganda 41 per 1,000 births (eight times greater than the HAPO study ${ }^{13,30}$ ), but we observed a lower than expected figure of 28 per 1,000 births - perhaps because our study was biased towards the urban population.

Mean arterial pressure (MAP) $>90 \mathrm{mmHg}$ (equivalent to blood pressure of $>130 / 85 \mathrm{mmHg}$ ) was the strongest predictor of perinatal mortality. High mid-trimester MAP may be indicative of pre-existing hypertension, or evolving gestational hypertension or preterm pre-eclampsia. The strong association between such hypertensive disorders and perinatal mortality in this setting is well recognised. ${ }^{31}$ High blood pressure is linked to other determinants such as maternal age, BMI and hyperglycaemia. Indeed GDM is associated with a higher risk of hypertensive disorders, and treating GDM might have benefit of reducing the incidence. ${ }^{6}$ However, in our study, the significant contribution of MAP to perinatal mortality was independent of GDM diagnosis, maternal age, and mid-trimester BMI. This supports identification of high blood pressure as a key strategy to reduce stillbirth and neonatal death.

Of our secondary outcomes, there was a significant association between GDM, glucose concentrations and Caesarean delivery. We did not have the depth of data to conclude whether clinicians were more likely to intervene with Caesarean delivery merely from the diagnosis of GDM, ${ }^{27,32}$ or due to true clinical indication from obstructed labour or neonatal issues. Three quarters of Caesarean deliveries were coded as 'emergency' rather than 'elective' in our study, which may suggest suboptimal delivery planning. This warrants further study as any unindicated Caesarean section in the low-resource setting carries undue risk, particularly when performed as emergencies. ${ }^{19}$ There was a tendency for a positive association between fasting glucose and c-peptide concentration in umbilical cord blood. This is in accord with previous data, including from the HAPO study, that have shown that maternal fasting glucose is a strong predictor of umbilical cord c-peptide concentration and clinical neonatal hypoglycaemia. ${ }^{3}$ The difference in reported neonatal hypoglycaemia between the GDM and normoglycaemic groups in our study is limited by the predilection to test babies born to mothers with a label of GDM. The rarity of the outcome reflects the challenges faced in this setting to detect neonatal hypoglycaemia which offers a potential argument in favour of testing for and managing GDM if the association with high cord c-peptide is true. However, no reduction in neonatal hypoglycaemia was detected in either of the large trials assessing treatment of GDM, even in the context of management strategies that would nonetheless be unfeasible in our setting. ${ }^{6,7}$

Conclusion (to include practical and research recommendations)

Our data from Uganda indicate that GDM, even when not optimally managed, is not associated with significant short term adverse outcomes, notably excessive birthweight or perinatal death. Instead, simple screening and interventions to manage obesity and hypertension may be more important in reducing adverse pregnancy outcomes. However, more studies will also be required to examine the effect of GDM beyond immediate peripartum complications, as HIP has been implicated in fetal programming - with potential to increase lifetime cardiovascular risk for both mother and baby. ${ }^{33,34}$

Acknowledgements 
Many thanks to Dr Kate Wiles and Dr Jane Hirst for your expert and invaluable contribution to the development of the manuscript.

Disclosure of interests

The authors declare no conflicts of interest

Contribution of authorship

JM and BNak made an equal contribution to the overall preparation of the final manuscript. JM, BNak and BNat were responsible for the statistical analysis plan. MJN was responsible for the overall conception and planning of the study. BNat, IS, MM and EW were primarily responsible for data management and statistical analysis. All authors (JM, BNak, BNat, IS, MM, AAN, STu, STi, MM, AK, BAg, FN, DZ, BAy, HK, SN, MS, AN, EW and MN) were responsible for carrying out the study, contributing to the final manuscript and accept responsibility for the published paper.

Details of ethics approval

This research project was approved by the research and ethics committee of the Uganda Virus Research Institute (approval GC/127/19/04/625) and the Uganda National Council for Science and Technology (approval HS2340).

\section{Funding}

The study was funded by an MRC strategic award granted to Professor Moffat Nyirenda

\section{REFERENCES}

1. Diagnostic Criteria and Classification of Hyperglycaemia First Detected in Pregnancy [Internet]. Geneva: World Health Organization; 2013 [cited 2020 Mar 4]. (WHO Guidelines Approved by the Guidelines Review Committee). Available from: http://www.ncbi.nlm.nih.gov/books/NBK169024/

2. Farrell T, Neale L, Cundy T. Congenital Anomalies in the Offspring of Women With Type 1, Type 2 and Gestational Diabetes: Obstetrical \& Gynecological Survey [Internet]. 2002 Oct [cited 2020 Mar 4];57(10):647-8. Available from: http://journals.lww.com/00006254-200210000-00002

3. Hyperglycemia and Adverse Pregnancy Outcomes. N Engl J Med [Internet]. 2008 May 8 [cited 2020 Mar 4];358(19):1991-2002. Available from: http://www.nejm.org/doi/abs/10.1056/NEJMoa0707943

4. Coustan DR, Lowe LP, Metzger BE, Dyer AR, International Association of Diabetes and Pregnancy Study Groups. The Hyperglycemia and Adverse Pregnancy Outcome (HAPO) study: paving the way for new diagnostic criteria for gestational diabetes mellitus. Am J Obstet Gynecol. 2010 Jun;202(6):654.e1-6.

5. Kibirige D, Milln J, Nyirenda MJ. Availability and Affordability of Essential Medicines and Diagnostic Tests for Diabetes Mellitus and Cardiovascular Diseases in Sub-Saharan Africa: A Systematic Review SSRN: 3259477. Available from: https://ssrn.com/abstract $=3259477$

6. Crowther CA, Hiller JE, Moss JR, McPhee AJ, Jeffries WS, Robinson JS. Effect of Treatment of Gestational Diabetes Mellitus on Pregnancy Outcomes. N Engl J Med [Internet]. 2005 Jun 16 [cited 2020 Mar 4];352(24):2477-86. Available from: http://www.nejm.org/doi/abs/10.1056/NEJMoa042973

7. Landon MB, Spong CY, Thom E, Carpenter MW, Ramin SM, Casey B, et al. A multicenter, randomized trial of treatment for mild gestational diabetes. N Engl J Med. 2009 Oct 1;361(14):1339-48.

8. International Diabetes Federation. IDF Diabetes Atlas 2019 [Internet]. Available from: https://www.diabetesatlas.org/upload/resources/material/20200302_133351_IDFATLAS9e-final-web.pdf

9. Nakabuye B, Bahendeka S, Byaruhanga R. Prevalence of hyperglycaemia first detected during pregnancy and subsequent obstetric outcomes at St. Francis Hospital Nsam- 
bya. BMC Res Notes [Internet]. 2017 Dec [cited 2020 Mar 4];10(1):174. Available from: http://bmcresnotes.biomedcentral.com/articles/10.1186/s13104-017-2493-0

10. Macaulay S, Dunger DB, Norris SA. Gestational Diabetes Mellitus in Africa: A Systematic Review. Schillaci G, editor. PLoS ONE [Internet]. 2014 Jun 3 [cited 2019 Nov 5];9(6):e97871. Available from: https://dx.plos.org/10.1371/journal.pone.0097871

11. Natamba BK, Namara AA, Nyirenda MJ. Burden, risk factors and maternal and offspring outcomes of gestational diabetes mellitus (GDM) in sub-Saharan Africa (SSA): a systematic review and metaanalysis. BMC Pregnancy Childbirth [Internet]. 2019 Dec [cited 2020 Mar 4];19(1):450. Available from: https://bmcpregnancychildbirth.biomedcentral.com/articles/10.1186/s12884-019-2593-z

12. Uganda GDP World Bank [Internet]. Available from: https://data.worldbank.org/indicator/NY.GDP.PCAP.CD?location

13. WHO. WHO data; maternal,newborn, child and adolescent health. [Internet]. Available from: https://www.who.int/data/maternal-newborn-child-adolescent/maternal-and-newborn-data/maternal-andnewborn - mortality-causes-of-death/mca/maternal-and-newborn — mortality-causes-of-death

14. Uganda Demographic and Health Survey 2016 [Internet]. Available from: https://www.dhsprogram.com/publications/publication-fr333-dhs-final-reports.cfm

15. Hod M, Kapur A, Sacks DA, Hadar E, Agarwal M, Di Renzo GC, et al. The International Federation of Gynecology and Obstetrics (FIGO) Initiative on gestational diabetes mellitus: A pragmatic guide for diagnosis, management, and care \#. International Journal of Gynecology \& Obstetrics [Internet]. 2015 Oct [cited 2020 Mar 8];131:S173-211. Available from: http://doi.wiley.com/10.1016/S0020-7292\%2815\%2930033-3

16. Intergrowth 21 - Standards and Tools [Internet]. Available from: https://intergrowth21.tghn.org/standards-tools/

17. The World Bank data [Internet]. Available from: https://data.worldbank.org/?locations=UG-GB

18. Marseille E, Lohse N, Jiwani A, Hod M, Seshiah V, Yajnik CS, et al. The cost-effectiveness of gestational diabetes screening including prevention of type 2 diabetes: application of a new model in India and Israel. The Journal of Maternal-Fetal \& Neonatal Medicine [Internet]. 2013 May [cited 2020 Mar 8];26(8):802-10. Available from: http://www.tandfonline.com/doi/full/10.3109/14767058.2013.765845

19. Sobhy S, Arroyo-Manzano D, Murugesu N, Karthikeyan G, Kumar V, Kaur I, et al. Maternal and perinatal mortality and complications associated with caesarean section in low-income and middle-income countries: a systematic review and meta-analysis. The Lancet [Internet]. 2019 May [cited 2020 Mar 3];393(10184):197382. Available from: https://linkinghub.elsevier.com/retrieve/pii/S0140673618323869

20. Kyei-Nimakoh M, Carolan-Olah M, McCann TV. Access barriers to obstetric care at health facilities in sub-Saharan Africa-a systematic review. Syst Rev. 2017 06;6(1):110.

21. Handa VL, Lockhart ME, Fielding JR, Bradley CS, Brubaker L, Cundiff GW, et al. Racial Differences in Pelvic Anatomy by Magnetic Resonance Imaging: Obstetrics \& Gynecology [Internet]. 2008 Apr [cited 2020 Mar 4];111(4):914-20. Available from: http://content.wkhealth.com/linkback/openurl?sid=WKPTLP:landingpage\&an=00006250-20080400000016

22. Baragi RV, DeLancey JOL, Caspari R, Howard DH, Ashton-Miller JA. Differences in pelvic floor area between African American and European American women. American Journal of Obstetrics and Gynecology [Internet]. 2002 Jul [cited 2020 Mar 4];187(1):111-5. Available from: https://linkinghub.elsevier.com/retrieve/pii/S0002937802000911

23. Dewey KG, Begum K. Long-term consequences of stunting in early life: Long-term consequences of stunting. Maternal \& Child Nutrition [Internet]. 2011 Oct [cited 2020 Mar 4];7:5-18. Available from: http://doi.wiley.com/10.1111/j.1740-8709.2011.00349.x 
24. Munabi IG, Luboga SA, Luboobi L, Mirembe F. Association between Maternal Pelvis Height and Intrapartum Foetal Head Moulding in Ugandan Mothers with Spontaneous Vertex Deliveries. Obstetrics and Gynecology International [Internet]. 2016 [cited 2020 Mar 4];2016:1-7. Available from: http://www.hindawi.com/journals/ogi/2016/3815295/

25. Pepe MS. Limitations of the Odds Ratio in Gauging the Performance of a Diagnostic, Prognostic, or Screening Marker. American Journal of Epidemiology [Internet]. 2004 May 1 [cited 2020 Mar 3];159(9):88290. Available from: https://academic.oup.com/aje/article-lookup/doi/10.1093/aje/kwh101

26. HAPO Study Cooperative Research Group. Hyperglycaemia and Adverse Pregnancy Outcome (HAPO) Study: associations with maternal body mass index: HAPO - BMI and perinatal outcomes. BJOG: An International Journal of Obstetrics \& Gynaecology [Internet]. 2010 Apr [cited 2020 Mar 8];117(5):575-84. Available from: http://doi.wiley.com/10.1111/j.1471-0528.2009.02486.x

27. Cundy T, Ackermann E, Ryan EA. Gestational diabetes: new criteria may triple the prevalence but effect on outcomes is unclear. BMJ [Internet]. 2014 Mar 11 [cited 2020 Mar 4];348(mar11 6):g1567-g1567. Available from: http://www.bmj.com/cgi/doi/10.1136/bmj.g1567

28. Kamanu CI, Onwere S, Chigbu B, Aluka C, Okoro O, Obasi M. Fetal macrosomia in African women: a study of 249 cases. Arch Gynecol Obstet [Internet]. 2009 Jun [cited 2020 Mar 8];279(6):857-61. Available from: http://link.springer.com/10.1007/s00404-008-0780-7

29. Ozumba BC, Obi SN, Oli JM. Diabetes mellitus in pregnancy in an African population. International Journal of Gynecology \& Obstetrics [Internet]. 2004 Feb [cited 2020 Mar 8];84(2):114-9. Available from: http://doi.wiley.com/10.1016/S0020-7292\%2803\%2900210-8

30. WHO Maternal mortality report 2000-2017 [Internet]. Available from: https://www.unfpa.org/sites/default/files/pub-pdf/Maternal_mortality_report.pdf

31. Kiondo P, Tumwesigye NM, Wandabwa J, Wamuyu-Maina G, Bimenya GS, Okong P. Adverse neonatal outcomes in women with pre-eclampsia in Mulago Hospital, Kampala, Uganda: a cross-sectional study. Pan Afr Med J. 2014;17 Suppl 1:7.

32. Coustan DR. Management of gestational diabetes mellitus: a self-fulfilling prophecy? JAMA. 1996 Apr 17;275(15):1199-200.

33. Fleming TP, Watkins AJ, Velazquez MA, Mathers JC, Prentice AM, Stephenson J, et al. Origins of lifetime health around the time of conception: causes and consequences. The Lancet [Internet]. 2018 May [cited 2020 Mar 4];391(10132):1842-52. Available from: https://linkinghub.elsevier.com/retrieve/pii/S014067361830312X

34. Nyirenda MJ, Byass P. Pregnancy, programming, and predisposition. The Lancet Global Health [Internet]. 2019 Apr [cited 2020 Mar 4];7(4):e404-5. Available from: https://linkinghub.elsevier.com/retrieve/pii/S2214109X19300518

\section{Supplementary Appendix}

Table S1 insert here.

Table S2 insert here.

Table S3 insert here.

Table S4 insert here.

Table S5 insert here.

\section{Hosted file}


Fig1_GDM Africa_BJOG.docx available at https://authorea.com/users/320761/articles/450181gestational-diabetes-gdm-does-not-predict-large-birthweight-or-perinatal-death-in-arelatively-untreated-population-in-uganda-a-prospective-observational-cohort-study

\section{Hosted file}

Table1_GDM Africa_BJOG.docx available at https://authorea.com/users/320761/articles/450181gestational-diabetes-gdm-does-not-predict-large-birthweight-or-perinatal-death-in-arelatively-untreated-population-in-uganda-a-prospective-observational-cohort-study

\section{Hosted file}

Table2_GDM Africa_BJOG.docx available at https://authorea.com/users/320761/articles/450181gestational-diabetes-gdm-does-not-predict-large-birthweight-or-perinatal-death-in-arelatively-untreated-population-in-uganda-a-prospective-observational-cohort-study

\section{Hosted file}

Table3_GDM Africa_BJOG.docx available at https://authorea.com/users/320761/articles/450181gestational-diabetes-gdm-does-not-predict-large-birthweight-or-perinatal-death-in-arelatively-untreated-population-in-uganda-a-prospective-observational-cohort-study

\section{Hosted file}

TableS1_GDM Africa_BJOG.docx available at https://authorea.com/users/320761/articles/450181gestational-diabetes-gdm-does-not-predict-large-birthweight-or-perinatal-death-in-arelatively-untreated-population-in-uganda-a-prospective-observational-cohort-study

\section{Hosted file}

TableS2_GDM Africa_BJOG_chDIP.docx available at https://authorea.com/users/320761/articles/ 450181-gestational-diabetes-gdm-does-not-predict-large-birthweight-or-perinatal-deathin-a-relatively-untreated-population-in-uganda-a-prospective-observational-cohort-study

\section{Hosted file}

TableS3_GDM Africa_BJOG_outDIP.docx available at https://authorea.com/users/320761/articles/ 450181-gestational-diabetes-gdm-does-not-predict-large-birthweight-or-perinatal-deathin-a-relatively-untreated-population-in-uganda-a-prospective-observational-cohort-study

\section{Hosted file}

TableS4_GDM Africa_BJOG_mxGDM.docx available at https://authorea.com/users/320761/articles/ 450181-gestational-diabetes-gdm-does-not-predict-large-birthweight-or-perinatal-deathin-a-relatively-untreated-population-in-uganda-a-prospective-observational-cohort-study

\section{Hosted file}

TableS5_GDM Africa_BJOG.docx available at https://authorea.com/users/320761/articles/450181gestational-diabetes-gdm-does-not-predict-large-birthweight-or-perinatal-death-in-arelatively-untreated-population-in-uganda-a-prospective-observational-cohort-study 